\title{
A comprehensive analysis of precursor microRNA cleavage by human Dicer
}

\author{
YONG FENG, ${ }^{1,2,4}$ XIAOXIAO ZHANG, ${ }^{1,4}$ PAUL GRAVES, ${ }^{3}$ and YAN ZENG ${ }^{1,5}$ \\ ${ }^{1}$ Department of Pharmacology, University of Minnesota, Minneapolis, Minnesota 55455, USA \\ ${ }^{2}$ Department of Medical Microbiology, School of Basic Medical Sciences, Wuhan University, Wuhan, Hubei 430070, P.R. China \\ ${ }^{3}$ Department of Radiation Oncology, New York Methodist Hospital, Weill Cornell Medical College, Brooklyn, New York 11215, USA
}

\begin{abstract}
Dicer cleaves double-stranded RNAs (dsRNAs) or precursor microRNAs (pre-miRNAs) to yield 22-nt RNA duplexes. The premiRNA structure requirement for human Dicer activity is incompletely understood. By large-scale in vitro dicing assays and mutagenesis studies, we showed that human Dicer cleaves most, although not all, of the 161 tested human pre-miRNAs efficiently. The stable association of RNAs with Dicer, as examined by gel shift assays, appears important but is not sufficient for cleavage. Human Dicer tolerates remarkable structural variation in its pre-miRNA substrates, although the dsRNA feature in the stem region and the 2-nt 3 '-overhang structure in a pre-miRNA contribute to its binding and cleavage by Dicer, and a large terminal loop further enhances pre-miRNA cleavage. Dicer binding protects the terminal loop from digestion by S1 nuclease, suggesting that Dicer interacts directly with the terminal loop region.
\end{abstract}

Keywords: Dicer; pre-miRNA; RNA binding; RNA processing; secondary structure

\section{INTRODUCTION}

miRNAs are a family of small RNAs that regulate gene expression post-transcriptionally in eukaryotes (Bartel 2004). In mammalian cells, a miRNA gene is initially transcribed to produce a long, primary miRNA transcript (pri-miRNA), which is then processed into an $\sim 60$ - to 70-nt hairpin structured pre-miRNA by the Drosha/DGCR8 holoenzyme. Another RNase, Dicer, cleaves the pre-miRNA to generate an $\sim 22$-nt-long miRNA duplex. Dicer also cleaves extended dsRNAs to produce small interfering RNA duplexes. Finally, an Argonaute protein will select one of the strands to form an RNA:protein complex, which subsequently represses target gene expression.

Dicer is highly conserved and expressed in both unicellular and multicellular organisms. Dicer in Giardia intestinalis forms a "hatchet" shape, with its PAZ domain binding to the 2-nt $3^{\prime}$ overhang of a dsRNA, and the RNase III domains cleaving $\sim 65 \AA$ or 25 bp away from the $3^{\prime}$ end (MacRae et al. 2006). In humans, the end structures or sequences of RNA substrates also influence the processing

\footnotetext{
${ }^{4}$ These authors contributed equally to this work.

${ }^{5}$ Corresponding author

E-mail zengx033@umn.edu

Article published online ahead of print. Article and publication date are at http://www.rnajournal.org/cgi/doi/10.1261/rna.033688.112.
}

specificity and efficiency by Dicer (Vermeulen et al. 2005; Park et al. 2011). Human Dicer (hDicer) recognizes the $5^{\prime}$ end and/or the 2-nt $3^{\prime}$ overhang in a pre-miRNA or dsRNA and cleaves $\sim 22$ nt into the stem (Park et al. 2011). In addition, cleavage by hDicer is negatively affected by an increased base-pairing feature in the stem region ( $\mathrm{Ma}$ et al. 2008; Chakravarthy et al. 2010; Zhang and Zeng 2010) or a small terminal loop in pre-miRNAs (Zhang and Zeng 2010). Drosophila melanogaster expresses two Dicer isoforms, with Dicer-1 (Dcr-1) being responsible for the processing of pre-miRNAs, and Dicer-2 for long dsRNAs (Lee et al. 2004). Tsutsumi et al. (2011) reported that Dcr-1 selects the correct substrates by measuring the distance between the terminal loop and the 2-nt $3^{\prime}$ overhang of a pre-miRNA. Extending the stem region or reducing the loop size compromised the processing by Dcr-1, seemingly consistent with the findings with hDicer (Ma et al. 2008; Chakravarthy et al. 2010; Zhang and Zeng 2010), even though the same study also showed that hDicer did not discriminate against such different RNA substrates (Tsutsumi et al. 2011). In addition, while Dcr-1's substrate binding affinity, as measured by gel shift assays, primarily determines RNA cleavage efficiencies (Tsutsumi et al. 2011), Vermeulen et al. (2005) and Chakravarthy et al. (2010) showed that differences in RNA cleavage by hDicer were not simply a consequence of substrate binding. 
The discrepancies in the above, reported substrate preferences of hDicer and Dcr-1 may be due to genuine differences between the two enzymes, different assay conditions, and/or different substrates used in the studies. Previous work examined a small number of arbitrarily selected pre-miRNAs and their mutants as Dicer substrates (e.g., $\mathrm{Ma}$ et al. 2008; Chakravarthy et al. 2010; Zhang and Zeng 2010; Park et al. 2011; Starega-Roslan et al. 2011; Tsutsumi et al. 2011). The human genome encodes hundreds of miRNA genes, and the predicted pre-miRNAs vary greatly in the end structure, stability and length of the duplex region, and size of the terminal loop. How hDicer binds and cleaves these diverse substrates has not been examined systematically. In this study, we performed in vitro hDicer cleavage and binding assays on a large number of predicted, human pre-miRNAs and several series of the pre-miRNA mutants, in order to determine comprehensively how pre-miRNA structural features impact hDicer function. Moreover, our previous study showed that Drosha cleaves human primiRNAs differentially, which may be a general mechanism to regulate mature miRNA expression in vivo (Feng et al. 2011). It would be interesting to determine if Dicer exhibits the analogous substrate preferences and to compare the relative contribution by Drosha and Dicer to differential miRNA expression.

\section{RESULTS}

\section{Cleavage of pre-miRNAs by hDicer in vitro}

For our studies of hDicer function, we first overexpressed and purified recombinant hDicer from $293 \mathrm{~T}$ cells. Figure 1A shows that the hDicer preparation contained predominantly a single protein of $\sim 200 \mathrm{kDa}$, expected for the fulllength Dicer. We did not overexpress Dicer cofactors such as TRBP because it has been shown that TRBP contributes little to Dicer substrate selectivity (Chakravarthy et al. 2010; Koscianska et al. 2011; Tsutsumi et al. 2011). RNA substrates were then prepared by in vitro transcription and labeled with ${ }^{32} \mathrm{P}$ as described in Materials and Methods. Preliminary studies indicated that hDicer cleaved internally labeled and 5'-labeled, select pre-miRNAs at similar rates as pre-miRNAs isolated from Drosha cleavage reactions of internally labeled pri-miRNAs (Zeng et al. 2005; data not shown). Furthermore, chemically synthesized RNAs were cleaved identically as the in vitro-transcribed RNAs (see Fig. $4 \mathrm{C}, \mathrm{D}$ below). As a result, we used the simplest, internally labeled, in vitro-transcribed RNAs as substrates in most of the subsequent hDicer processing reactions, unless indicated otherwise.

We previously showed that human pri-miRNAs vary greatly in their Drosha susceptibility in vitro (Feng et al. 2011). For this study, we examined the 111 pre-miRNAs whose pri-miRNAs were detectably cleaved by Drosha; in addition, we randomly selected 50 pre-miRNAs whose primiRNAs were not cleaved by Drosha (Feng et al. 2011). Together these pre-miRNAs were subjected to hDicer processing in vitro. First, in a time-course study, we incubated pre-let-7a-1 (pre-let-7a in short), pre-miR-892a, and premiR-491 with a molar excess of hDicer and removed aliquots of the reactions at 5,10,20,40, and $60 \mathrm{~min}$ for analysis. As shown in Figure 1B and quantified in Figure 1C, hDicer cleavage products continued to accumulate up to $60 \mathrm{~min}$, and the differences in cleavage efficiencies of the premiRNAs were largely constant during the period. These results suggested that single time-point assays could faithfully reveal the differences in hDicer cleavage of pre-miRNAs, which facilitated our survey of the 161 pre-miRNAs in vitro. We further included pre-let-7a in every processing experiment as a normalization control so that the relative cleavage efficiencies of all of the other pre-miRNAs could be compared with that of pre-let-7a, which was set at 100 .

Under the condition in which hDicer $(\sim 1 \mathrm{nM})$ was in excess over pre-miRNAs $(\sim 0.05-0.2 \mathrm{nM})$, most of the 161 pre-miRNAs were well digested by hDicer (Fig. 2A; data summarized in Supplemental Table 1). We subjected more than 50 pre-miRNAs to replicate end-point and/or timecourse assays with varying amounts of hDicer and RNAs, 


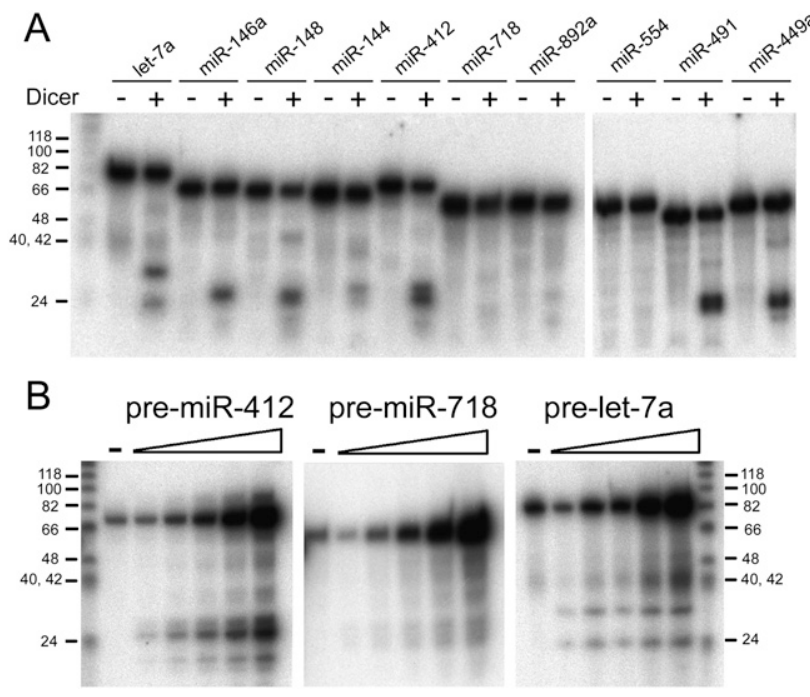

FIGURE 2. Differential pre-miRNA cleavage by hDicer. (A) Examples of end-point analysis of pre-miRNA cleavage by hDicer. PremiRNAs were incubated with hDicer for $\sim 60 \mathrm{~min}$. Sizes of DNA markers (in nucleotides) are indicated at the left. Shown are the representative results of at least three replicate experiments. $(B)$ Increasing amounts (0.1-6 nM) of pre-miR-412, pre-miR-718, and pre-let-7a were incubated with $0.2 \mathrm{nM}$ hDicer for $5 \mathrm{~min}$. Sizes of DNA markers (in nucleotides) are indicated. (-) Without hDicer addition. Shown are the representative results of at least three independent experiments.

which yielded reproducible results (data not shown). PremiRNAs still varied in their relative cleavage efficiencies, and a small number of them, such as pre-miR-188, premiR-554, pre-miR-569, pre-miR-718, and pre-miR-892a, were very poor $\mathrm{hDicer}$ substrates compared with pre-let-7a (Figs. 1B,C, 2A; Supplemental Table 1). For these premiRNAs we rechecked their sequences and predicted secondary structures in the miRBase and Mfold (Zuker 2003), repeated dicing assays, and confirmed that they were, indeed, only weakly digested by hDicer (see below; data not shown).

We also performed Michaelis-Menton analysis for select pre-miRNAs, with increasing amounts of pre-miRNAs incubated with hDicer (Fig. 2B). Pre-miRNAs diverged in their $K_{\mathrm{m}}$ and $V_{\max }: V_{\max }$ for pre-miR-412 was $5.1 \pm 1.1$ $\mathrm{nM} / \mathrm{min}$, pre-miR-718 was $0.2 \pm 0.1 \mathrm{nM} / \mathrm{min}$, and pre-let$7 \mathrm{a}$ was $0.6 \pm 0.1 \mathrm{nM} / \mathrm{min}$. $K_{\mathrm{m}}$ was $12 \pm 4 \mathrm{nM}$ for pre-miR$412,4 \pm 2 \mathrm{nM}$ for pre-miR-718, and $4 \pm 1 \mathrm{nM}$ for pre-let-7a. The values for pre-let-7a are of the same order of magnitude as those reported in the literature ( $\mathrm{Ma}$ et al. 2008; Chakravarthy et al. 2010). These results confirmed that hDicer discriminated against its pre-miRNA substrates-hence the relative reactivity. They further suggested that the differences could lie in how effectively hDicer interacts with RNAs as well as how fast it catalyzes the cleavage reactions.

To evaluate how the differences in pre-miRNA cleavage by hDicer might contribute to differential miRNA expressions in vivo, we computed the Spearman rank correlation between relative hDicer cleavage efficiencies and human miRNA expression (Landgraf et al. 2007). No significant correlation was detected $(\rho=0.13, p=0.11)$, while Drosha cleavage showed a significant, positive correlation with miRNA expression $(\rho=0.33, p<0.0001)$. When the values of Dicer cleavage efficiencies multiplied by Drosha cleavage efficiencies were correlated to miRNA expression, we again observed a significant correlation $(\rho=0.32, p=0.0001)$. It appeared, therefore, that substrate discrimination by Drosha plays a larger role in regulating differential miRNA expression in humans.

\section{Complex formation between hDicer and pre-miRNAs}

Why do dicing rates vary with pre-miRNAs? The easiest explanation is that hDicer binds pre-miRNA substrates preferentially. It has been reported that substrate binding affinity correlates well with cleavage efficiency by fly Dcr-1 (Tsutsumi et al. 2011), but not hDicer (Vermeulen et al. 2005; Chakravarthy et al. 2010). It should be noted, however, that only a handful of dsRNAs including pre-miRNAs have been examined for Dicer binding. To evaluate how much binding affinity could explain dicing efficiency in vitro, therefore, we performed gel shift assays on a large number of pre-miRNAs to examine their interaction with hDicer (Fig. 3).

Incubating pre-miRNAs with increasing concentrations of hDicer led to increased RNA:hDicer complex formation in gel shift assays (Fig. $3 \mathrm{~A}, \mathrm{~B}$ ). From Figure $3 \mathrm{~A}$ and assuming 1:1 binding stoichiometry, the $K_{\mathrm{d}}$ for pre-let-7a was estimated to be $12 \pm 5 \mathrm{nM}$, which is of the same order of magnitude as reported (Ma et al. 2008), while $K_{\mathrm{d}}$ for premiR-412 was larger than $20 \mathrm{nM}$. That the $K_{\mathrm{d}}$ of pre-let-7a was smaller than that of pre-miR-412 is consistent with the comparison of their $K_{\mathrm{m}}$ values (Fig. $2 \mathrm{~B}$ ). $K_{\mathrm{d}}$ values are bigger than $K_{\mathrm{m}}$ likely because they were measured under different conditions: $K_{\mathrm{d}}$ by gel shift assays, $K_{\mathrm{m}}$ in solution. Figure 3, A and B, shows that hDicer associated with premiRNAs differentially, which is further demonstrated in Figure $3 \mathrm{C}$, in which a larger number of pre-miRNAs were incubated with a single concentration of hDicer. For the pre-miRNAs examined in Figure 3C, their relative hDicer binding and cleavage efficiencies are summarized in Figure 3D for comparisons. The extent of hDicer:pre-miRNA complex formation, as revealed by gel shift assays, could not fully explain differential dicing activities. For example, pre-miR-554 was a poorer substrate and bound hDicer more weakly than pre-let-7a (Fig. 3B-D), pre-miR-718 bound reasonably well to hDicer but had a very low dicing efficiency (Figs. 2A,B, 3B-D), whereas the opposite was true for pre-miR-412 (Figs. 2A,B, 3A,B). We concluded, therefore, that feature(s) in pre-miRNAs that did not contribute significantly to stable hDicer binding, as examined by a gel shift assay, might regulate the cleavage reaction as well. 


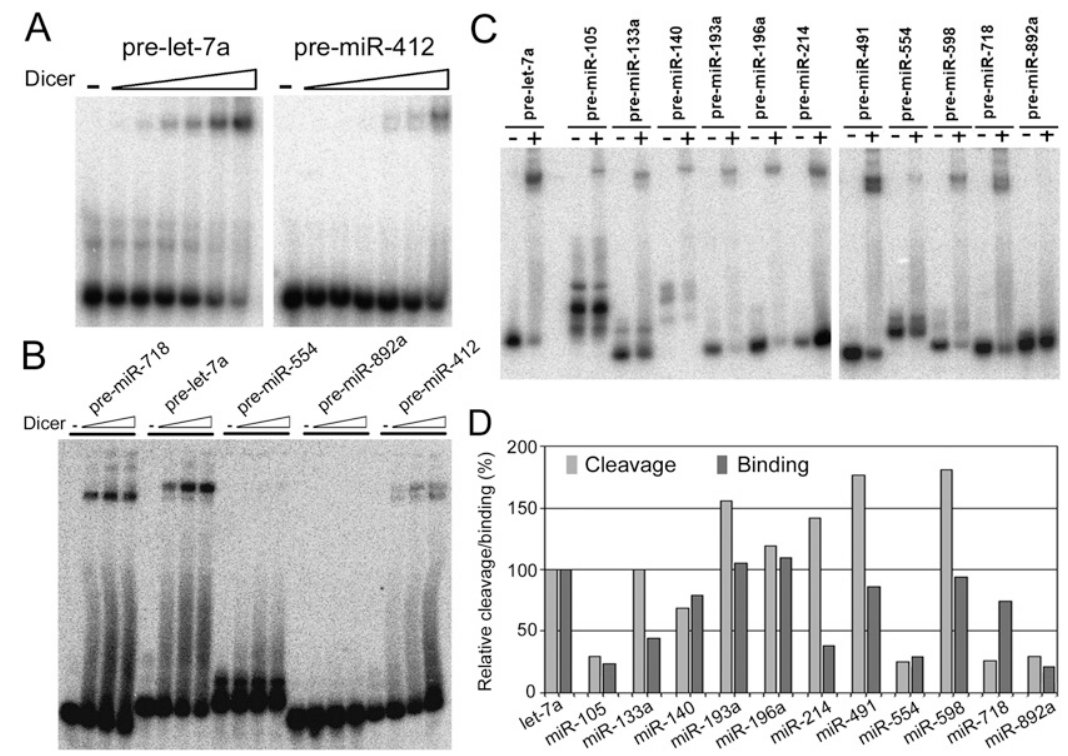

FIGURE 3. Gel shift assays to analyze hDicer:pre-miRNA interaction. (A) Increasing amounts of hDicer $(0,0.1,0.4,1,3,7$, and $20 \mathrm{nM})$ were incubated with $\sim 0.3 \mathrm{nM}^{32} \mathrm{P}$-labeled pre-let-7a or pre-miR-412 on ice for $40 \mathrm{~min}$, before fractionation on a $7.5 \%$ native gel. $(B)$ Increasing amounts of hDicer were incubated with the indicated pre-miRNAs. $(C)$ hDicer was incubated ("+") with the indicated pre-miRNAs before gel electrophoresis. (D) Comparisons of the relative hDicer cleavage efficiencies and binding efficiencies for the pre-miRNAs listed in $C$. Averaged percentages from at least two replicate tests are shown. For hDicer cleavage efficiencies, see Supplemental Table 1. Relative hDicer binding efficiency of a pre-miRNA was calculated by normalizing its binding to that of pre-let- $7 \mathrm{a}$, which was set at $100 \%$.

\section{The end structure of pre-miRNAs influences hDicer binding and cleavage}

While it is commonly assumed that pre-miRNAs contain a base-paired 5' end and a 2-nt 3' overhang per Drosha's RNase III activity, it is clear that human pre-miRNAs differ in their predicted end structures and that hDicer can cleave dsRNAs with variable ends (Zhang et al. 2002, 2004; Vermeulen et al. 2005; Kozomara and Griffiths-Jones 2011; Park et al. 2011). In fact, fewer than half of the pre-miRNA substrates tested in this study contained a predicted, paired $5^{\prime}$ end and a 2-nt $3^{\prime}$ overhang, and they were not, as a group, cleaved more efficiently than the rest of the premiRNAs, which typically contained both a $5^{\prime}$ and a $3^{\prime}$ overhang (Supplemental Table 1; data not shown). For example, Figure $4 \mathrm{~A}$ shows that pre-miRNAs with a 2 -nt $3^{\prime}$ overhang (miR-22 and miR-554) were no more efficiently processed by hDicer than pre-miRNAs with both a $5^{\prime}$ and a 3 ' overhang (let-7a, let-146a, and miR-223). In a gel shift assay, pre-miR-22 bound hDicer slightly weaker than pre-let-7a or pre-miR-223 (Fig. 4B). Thus, a canonical 2-nt $3^{\prime}$ overhang with a paired $5^{\prime}$ end is not essential for hDicer binding or cleavage.

Nevertheless, it is probable that the effect of the 2-nt $3^{\prime}$ overhang above was obscured by other structural features in the pre-miRNAs and that the $3^{\prime}$ overhang fine-tunes processing by hDicer (Park et al. 2011). So we constructed a series of mutants of pre-miR-449a that has a native, 2-nt $3^{\prime}$ overhang (Fig. 4C, left side). We also chemically synthesized the wild-type pre-miR-449a and mutants (Fig. 4C, right side). RNAs transcribed in vitro might contain heterogeneity at their ends with unknown frequencies (Milligan et al. 1987), which can be evaluated with the chemically synthesized RNAs serving as markers. Importantly, the chemically synthesized pre-miR-449a contains a $5^{\prime} \mathrm{U}$ residue identical to the predicted pre-miRNA in vivo, allowing us to compare how well the in vitro-transcribed RNAs represented endogenous pre-miRNAs. Both classes of RNAs were ${ }^{32} \mathrm{P}$-labeled at their $5^{\prime}$ ends, gel-isolated, digested with hDicer, and then fractionated on a denaturing gel. The wild-type pre-miR-449a was processed much more readily than the M1, M3, and M4 mutants that contain a $5^{\prime}$ overhang or a blunt end (Fig. 4D, cf. lanes 2 and 10 with lanes 4,6,8,12,14,16), and there was little difference in the cleavage efficiency or migration pattern between the in vitro-transcribed RNAs (Fig. 4D, lanes 1-8) and the corresponding, chemically synthesized RNAs (Fig. 4D, lanes 9-16). hDicer cleavage of the M2 mutant was also compromised (data not shown). These results suggest that our in vitrotranscribed RNAs were likely of the expected length and sequences, and that the $5^{\prime}$ and $3^{\prime}$ heterogeneity was of low abundance and would not significantly affect our conclusions. To study hDicer binding, in vitro-transcribed premiR-449a and mutants were subjected to gel shift assays. hDicer still associated with the mutant RNAs without a 3' overhang, although at a reduced level than the wild type (Fig. 4E). The same conclusion was reached using chemically synthesized RNAs (data not shown). These data demonstrated that everything else being equal, a canonical 3' overhang significantly facilitates $\mathrm{hDicer}$ binding and processing of pre-miRNAs.

\section{The impact of the stem and the terminal loop on hDicer function}

A pre-miRNA is composed of the miRNA duplex intermediate moiety and a terminal loop region. The terminal loop region is further predicted by RNA folding programs to form a very short stem and a single-stranded terminal loop. Previously we showed that a prominent dsRNA feature in the duplex region and a small terminal loop both hindered hDicer processing of pre-miRNAs (Zhang and Zeng 2010). Tsutsumi et al. (2011) reached the same 
A

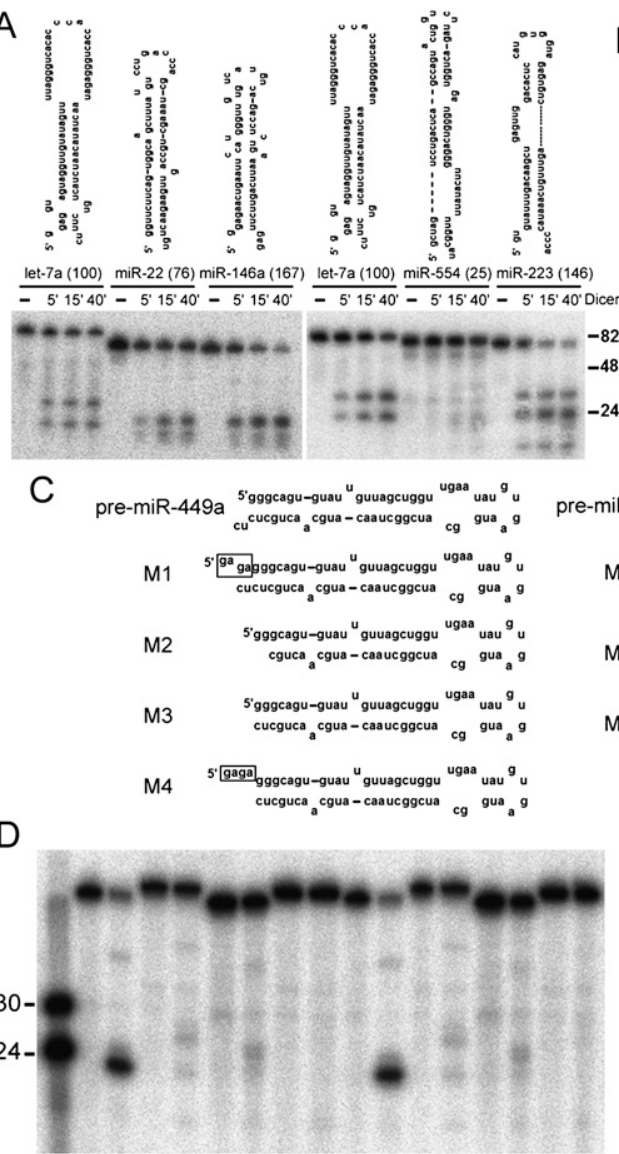

$\begin{array}{lllllllllllll}1 & 2 & 3 & 4 & 5 & 6 & 7 & 8 & 9 & 10 & 11 & 121314 & 1516\end{array}$
B

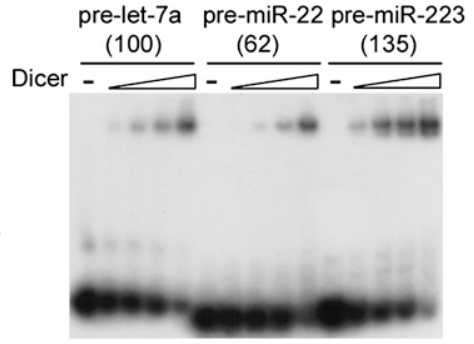

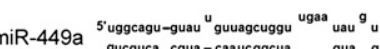
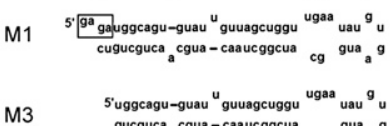

gucguca ${ }_{a}$ cgua - caaucggcua $\mathrm{cg}$ gua ${ }^{g}$
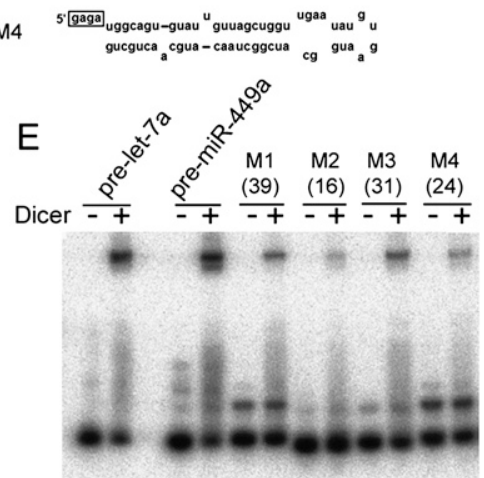

FIGURE 4. hDicer processing of pre-miRNAs with different end structures. (A) Pre-miRNAs with the predicted secondary structures shown on top were incubated with hDicer for 5,15 , and $40 \mathrm{~min}$. Sizes of DNA markers (in nucleotides) are indicated at the right. Relative hDicer cleavage efficiencies are in parentheses (Supplemental Table 1). (B) Gel shift assays with increasing amounts of hDicer incubated with the indicated pre-miRNAs. Relative hDicer binding efficiencies under the highest hDicer concentration are listed in parentheses. $(C)$ Predicted secondary structures of pre-miR-449a and its mutants. Shown on the left are the expected, in vitro transcribed RNAs, and on the right are the chemically synthesized RNAs. Added sequences are marked with a box. $(D)$ Dicing assay of RNAs in $C$. (Lanes $1,3,5,7,9,11,13,15)$ No hDicer; (lanes 2,4,6,8,10,12,14,16) incubated with hDicer; (lanes 1-8) in vitro transcribed RNAs as substrates; (lanes 9-16) chemically synthesized RNAs; (lanes 1,2,9,10) wild-type pre-miR-449a; (lanes 3,4,11,12) M1 mutant; (lanes 5,6,13,14) M3 mutant; (lanes 7,8,15,16) M4 mutant. RNA markers (in nucleotides) are shown at the left. (E) Gel shift assay. The indicated pre-miRNAs and mutants were incubated with hDicer on ice and then run on a native gel. Relative hDicer binding efficiencies in parentheses were calculated by normalizing to that of pre-miR-449a, which was set at 100 (data not shown). RNAs shown in $A, B$, and $E$ were tested at least twice. A representative result of three independent experiments is shown in $D$.

conclusion with the fly Dcr-1 but not with hDicer. To reconcile the differences, here, we first analyzed the relationship between terminal loop size and relative hDicer cleavage efficiency, based on the results of our large-scale dicing assays (Supplemental Table 1). Pre-miRNAs with a predicted loop size smaller than or equal to $3,4,5,6,7,8$, or $9 \mathrm{nt}$ were cleaved less efficiently than those of pre-miRNAs with a correspondingly larger loop (Table 1). Thus, a small terminal loop reduced hDicer cleavage of pre-miRNAs.
To further investigate how the stem and terminal loop characteristics affected hDicer activity, we constructed a series of mutants based on pre-miR-16-1 (pre-miR-16, in short), pre-miR-30a (pre-miR-30, in short), and pre-miR31 , with varying numbers of base pairs in the stem and differently sized terminal loops (Fig. 5A). Enforcing a perfect dsRNA feature in the stem region reduced cleavage by hDicer (Fig. 5B; e.g., cf. lane 2 with lanes 8 and 14, lane 16 with lanes 22 and 28 , lane 30 with lanes 36 and 42; summarized in Fig. 5D). Mutants with a 5- or 10-nt loop also had a lower dicing efficiency than the wild-type pre-miRNAs, which was partially rescued by installing a 14-nt loop (Fig. 5B; e.g., cf. lanes 2,4,6,8, lanes $16,18,20,22$, and lanes 30,38,42; Fig. 5D). Earlier studies had also showed that a natural pre-miRNA stem connected by a small terminal loop had modestly reduced hDicer cleavage as well (Zhang and Zeng 2010). We then compared how hDicer interacted with these RNAs by gel shift experiments. Interestingly, all the RNAs had a very similar degree of hDicer binding ability (Fig. 5C; data not shown). Figure 5D summarizes and compares the relative hDicer cleavage and binding efficiencies of the three premiRNAs and their mutants.

To gain insights into how hDicer binds these RNAs, we treated the hDicer:RNA complex with RNase V1, which digested dsRNA specifically. Preliminary studies showed that pre-miR-31 was less sensitive to RNase V1 (data not shown) than other RNAs, so we focused on pre-miR16 and pre-miR-30. hDicer binding, presumably to the dsRNA region, retarded RNase V1 digestion of the full-length substrates (Fig. 6A, cf. lanes 4 and 5 to 3 ), but the protection was less pronounced for the wild-type pre-miRNAs than for the indicated mutants. Under the same conditions, most of the RNAs would have been associated with hDicer, based on gel shift assays (data not shown), and hDicer did not significantly digest the RNAs (cf. lane 1 to lane 2). These results suggested that hDicer binds and protects the pre-miRNA stem, that the binding is dynamic, and that protection is stronger with a perfect stem.

We also used S1 nuclease to probe the interaction between hDicer and the single-stranded regions of the RNAs. As 
TABLE 1. Relationship between the terminal loop size of pre-miRNAs and hDicer cleavage efficiencies

\begin{tabular}{|c|c|c|c|c|c|}
\hline \multirow{2}{*}{$\begin{array}{l}\text { Loop } \\
\text { size, } \\
S(\mathrm{nt})\end{array}$} & \multicolumn{2}{|c|}{ Loop $\leq S$} & \multicolumn{2}{|c|}{ Loop $>S$} & \multirow[b]{2}{*}{$P$-value } \\
\hline & $\begin{array}{l}\text { Number of } \\
\text { pre-miRNAs }\end{array}$ & $\begin{array}{l}\text { Cleavage } \\
\text { efficiency }\end{array}$ & $\begin{array}{l}\text { Number of } \\
\text { pre-miRNAs }\end{array}$ & $\begin{array}{l}\text { Cleavage } \\
\text { efficiency }\end{array}$ & \\
\hline 3 & 3 & $49 \pm 14$ & 158 & $110 \pm 4$ & 0.02 \\
\hline 4 & 20 & $82 \pm 9$ & 141 & $112 \pm 4$ & 0.01 \\
\hline 5 & 33 & $84 \pm 7$ & 128 & $115 \pm 4$ & 0.002 \\
\hline 6 & 47 & $90 \pm 6$ & 114 & $116 \pm 5$ & 0.002 \\
\hline 7 & 58 & $95 \pm 6$ & 103 & $116 \pm 5$ & 0.007 \\
\hline 8 & 63 & $97 \pm 6$ & 98 & $116 \pm 5$ & 0.02 \\
\hline 9 & 78 & $98 \pm 5$ & 83 & $118 \pm 6$ & 0.01 \\
\hline 10 & 87 & $103 \pm 5$ & 74 & $115 \pm 6$ & 0.14 \\
\hline 11 & 100 & $106 \pm 5$ & 61 & $113 \pm 7$ & 0.46 \\
\hline 12 & 108 & $109 \pm 5$ & 53 & $108 \pm 7$ & 0.95 \\
\hline 13 & 124 & $108 \pm 4$ & 37 & $110 \pm 9$ & 0.82 \\
\hline 14 & 131 & $109 \pm 4$ & 30 & $105 \pm 10$ & 0.55 \\
\hline 15 & 140 & $110 \pm 4$ & 21 & $97 \pm 13$ & 0.19 \\
\hline 16 & 148 & $110 \pm 4$ & 13 & $88 \pm 9$ & 0.11 \\
\hline 17 & 153 & $110 \pm 4$ & 8 & $86 \pm 10$ & 0.12 \\
\hline
\end{tabular}

Shown are loop size $(S)$, the numbers of tested pre-miRNAs with a predicted loop $\leq S$ or $>S$, and their relative hDicer cleavage efficiencies (\%, average \pm standard deviations), according to the miRBase. The actual loop size could be variable and different. The Mann-Whitney U-test was used to calculate $P$-values.

shown in Figure 6, B and C, S1 predominantly cleaved at or near the terminal loop region, based on the size of cleavage products, but cleavage was greatly reduced in the presence of hDicer. hDicer did not cleave the RNAs (Fig. 6B; data not shown), and various pre-miRNA mutants were also shielded from S1 nuclease by hDicer (Fig. 6B,C). Thus, hDicer binding rendered the terminal loop region of hairpin RNAs resistant to S1 nuclease.

\section{Correcting pre-miRNA defects in hDicer binding or dicing}

As shown above, hDicer cleaved or bound some premiRNAs weakly. For example, pre-miR-892a bound hDicer more weakly than most other pre-miRNAs (Fig. 3B-D), while pre-miR-718 was a poor hDicer substrate but interacted relatively well with the protein (Figs. 2, 3). Based on our current knowledge, can we improve hDicer binding and/or cleavage of these pre-miRNAs? For pre-miR-892a, extending its stem region and creating a 2-nt $3^{\prime}$ overhang, as indicated in the M1 mutant, was predicted to enhance its binding to hDicer. This was indeed observed (Fig. 7A). Cleavage was modestly increased (data not shown).

Pre-miR-718 has a highly unstable stem and a 3-nt terminal loop and was cleaved poorly by hDicer (Fig. 2). Extending the stem (M1) had a small effect on dicing, but enlarging the loop (M2) significantly improved cleavage (Fig. 7B). Unexpectedly, both M1 and M2 exhibited reduced hDicer binding, compared with the wild-type pre-miRNA
(Fig. 7C), further underscoring the idea that hDicer cleavage and binding, as revealed by gel shift assays, can be uncoupled to a large degree.

\section{DISCUSSION}

Our current work started with a largely unbiased analysis of $\mathrm{hDicer}$ processing of 161 pre-miRNAs in vitro, at a much larger scale than previously reported. We found that most of them were good Dicer substrates, although their relative cleavage efficiencies still varied. When we compared the relative hDicer cleavage efficiencies with the published, human miRNA expression data (Landgraf et al. 2007), we did not observe a significant correlation. This result contrasts with what we obtained from the studies of pri-miRNA processing by human Drosha (Feng et al. 2011). Myriad confounding factors aside (Feng et al. 2011), a parsimonious explanation is that Drosha's action is more ratelimiting than Dicer's such that Drosha's relative specificity contributes more prominently to differential miRNA expression in vivo.

Previous studies of Dicer processing examined only a limited number of select, long dsRNAs and short hairpin RNAs including pre-miRNAs. How representative those pre-miRNA substrates were was unknown. A commonly accepted, "model" pre-miRNA has a helical 5' end, a 2-nt 3' overhang, an imperfect stem, and a terminal loop (Sashital and Doudna 2010), but complex genomes such as ours encode hundreds of miRNA genes, and the predicted pre-miRNAs differ substantially in their secondary structures. Our analysis subjected a much larger number of human pre-miRNAs to hDicer binding and cleavage assays, and the results conclusively demonstrate how individual RNA structural features contribute to hDicer activity.

Firstly, we showed that a canonical structure composed of a paired $5^{\prime}$ end and a 2-nt $3^{\prime}$ overhang is not essential but enhances hDicer binding and cleavage. Many premiRNAs possess unpaired residues at both their $5^{\prime}$ and $3^{\prime}$ ends and were well diced (e.g., Fig. 4A). This result is consistent with previous findings using dsRNAs (e.g., Provost et al. 2002; Zhang et al. 2002, 2004; Vermeulen et al. 2005; MacRae et al. 2007; Du et al. 2008; Welker et al. 2011) and pre-miRNAs as substrates (Park et al. 2011) and can be explained by hDicer having a pocket that recognizes the $5^{\prime}$ end of substrates (Park et al. 2011). On the other hand, removing the 2-nt $3^{\prime}$ overhang in the wild-type pre-miR449 a, by introducing a blunt end or $5^{\prime}$ overhangs, reduced hDicer processing (Fig. 4D) as well as binding (Fig. 4E). This is likely due to the presence of a PAZ domain in Dicer that preferentially binds a 2-nt 3' overhang (Ma et al. 2004). One caveat in our study is that for every miRNA gene, we tested only one pre-miRNA according to the most prevalent miRNA and miRNA* in miRBase. Because of the promiscuities in Drosha and Dicer cleavage sites (e.g., 

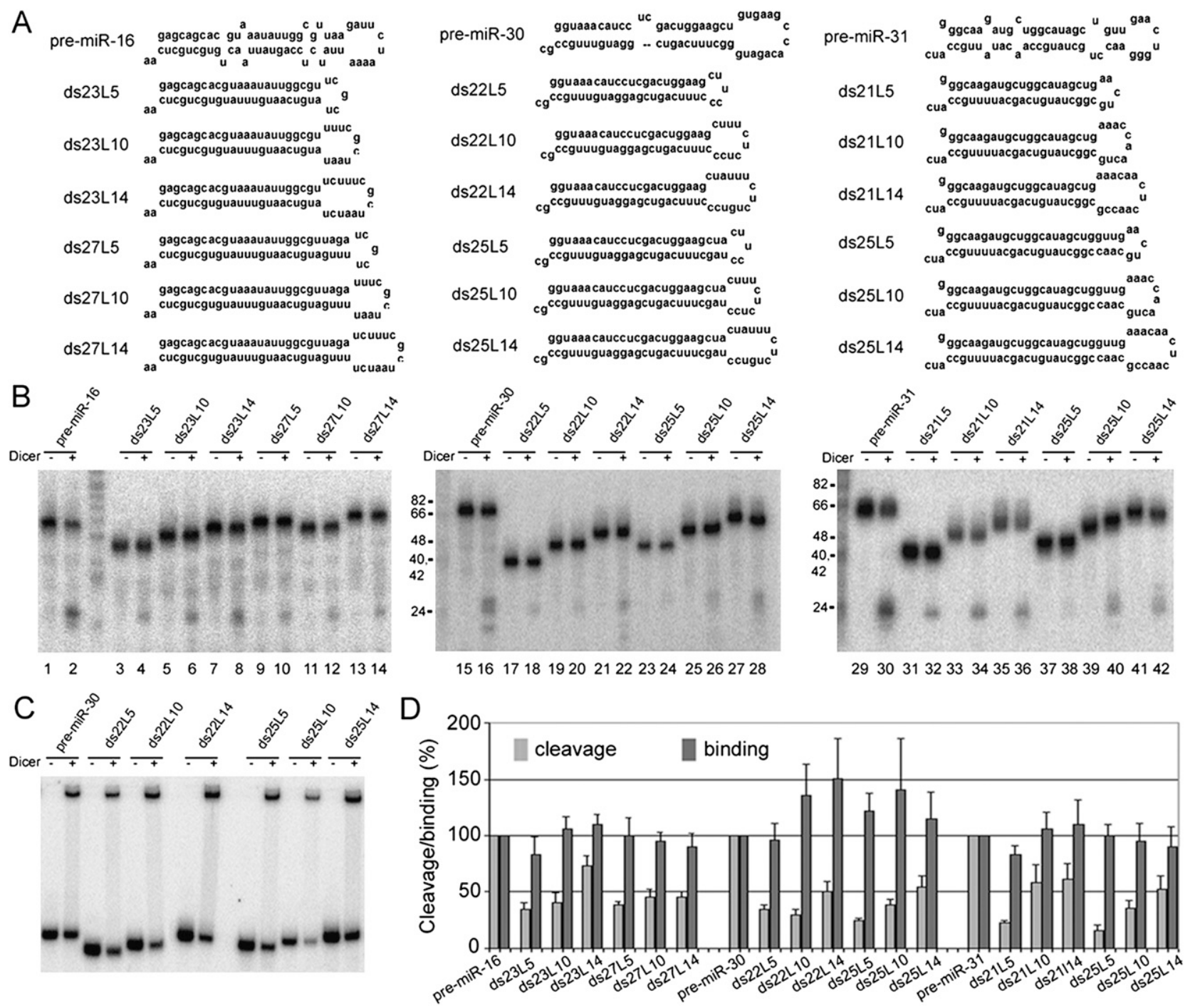

FIGURE 5. Impacts of the stem and terminal loop on hDicer function. (A) Predicted secondary structures of pre-miR-16, pre-miR-30, pre-miR31 , and their mutants. $(B)$ hDicer digestion of the pre-miRNAs and mutants. Sizes of DNA markers (in nucleotides) are indicated in the left. (C) Gel shift analysis of the binding of hDicer to the indicated pre-miR-30 and mutants. $(D)$ Comparison of the relative hDicer cleavage and binding efficiencies for the pre-miRNAs and mutants listed in $A$. For relative hDicer cleavage and binding efficiencies, the respective, wild-type premiRNAs are set as $100 \%$.

Starega-Roslan et al. 2011) and miRNA strand selection by Argonaute proteins, there could be multiple pre-miRNAs produced from a pri-miRNA.

Secondly, we demonstrated that too much of the dsRNA feature in a pre-miRNA stem is detrimental to hDicer processing. Pre-miRNA stems vary greatly in the number of base pairs, bulges, and gaps, but most of the tested premiRNAs still bound hDicer in vitro (Fig. 3). It appears that a short stretch of dsRNA can confer binding in a gel shift assay, even though hDicer cleavage requires a minimally sized stem. Further extending the number of base pairs in a pre-miRNA stem did not significantly enhance hDicer binding yet clearly reduced cleavage (Fig. 5). In the presence of hDicer, the dsRNA-specific RNase V1 digested the wild-type pre-miRNAs faster than mutants with more base pairs, suggesting that hDicer spends more time on the mutants and/or protects them better (Fig. 6A). Too much of a dsRNA characteristic might slow enzyme turnover or allow hDicer to "slide" along the stem, due to an increase in binding sites, without engaging in the presumably necessary conformational changes and catalysis.

Lastly, natural pre-miRNAs with a predicted, small terminal loop 3-9 nt long were cleaved less efficiently than those with a correspondingly larger loop (Table 1). Wild-type pre-miRNAs were better hDicer substrates than variants with a smaller loop (Fig. 5B; Zhang and Zeng 2010), and the weak dicing of pre-miR-718 was rescued by introducing an 11-nt-long terminal loop (Fig. 

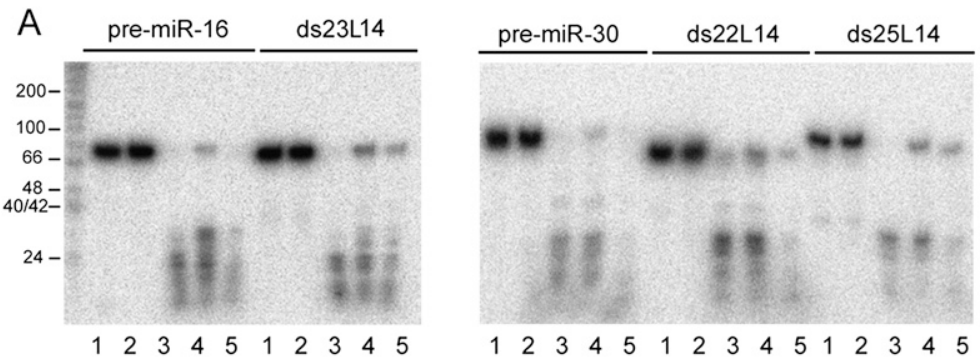

B

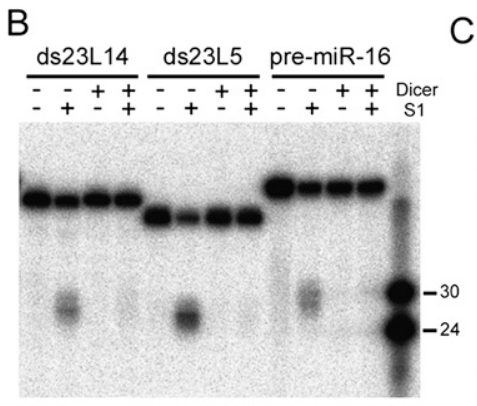

$\begin{array}{lllllllllllllll}1 & 2 & 3 & 4 & 5 & 1 & 2 & 3 & 4 & 5 & 1 & 2 & 3 & 4 & 5\end{array}$

C
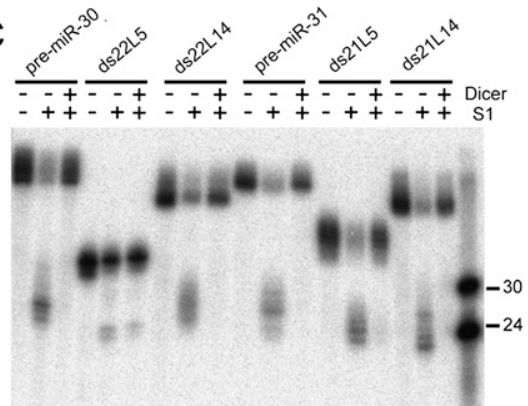

FIGURE 6. Nuclease protection assays to probe hDicer interactions with pre-miRNAs. (A) RNase V1 protection assays. (Lanes 1) $5^{\prime}$-end ${ }^{32} \mathrm{P}$-labeled RNA substrates without any treatment. (Lanes 2) RNAs treated with hDicer for $3 \mathrm{~min}$ at room temperature. (Lanes 3) RNAs treated with RNase V1 for 1 min at room temperature. (Lanes 4) RNAs treated with RNase V1 in the presence of hDicer for $1 \mathrm{~min}$ at room temperature. (Lanes 5) RNAs treated with RNase V1 in the presence of hDicer for $3 \mathrm{~min}$ at room temperature. Sizes of DNA markers are indicated at the left. (B) S1 nuclease cleavage of pre-miR-16 and the indicated mutants in the absence or presence of hDicer. RNA markers are indicated at the right. $(C)$ S1 nuclease cleavage of pre-miR-30, pre-miR-31, and their mutants, as in $B$. RNA markers are indicated at the right. Shown in $A-C$ are representatives of at least two experiments.

7B). Thus, both Drosha and Dicer prefer substrates with a large terminal loop, although the requirement by Drosha appears stricter (Zhang and Zeng 2010). A flexible terminal loop region also positively correlates with miRNA expression in vivo (Feng et al. 2011). Why is a large loop important, especially since Dicer can digest long dsRNAs without a terminal loop? There are at least two nonexclusive explanations. One is that Dicer may interact with and recognize the loop as a boundary. The other is that a small loop may introduce physical strains in the stem such that a "twisted" dsRNA is not well poised for cleavage. A small terminal loop seemed to negatively affect hDicer cleavage more than hDicer binding (Fig. 5B-D). Tsutsumi et al. (2011) proposed that the helicase domain of fly Dcr-1 recognizes the terminal loop, but the evidence was indirect. We found that the terminal loop was highly susceptible to S1 nuclease digestion, while the addition of hDicer largely protected it from S1 cleavage (Fig. 6B,C). Such physical evidence strongly suggests that $\mathrm{hDicer}$ binds the terminal loop.

$\mathrm{hDicer}$ binding, as examined by gel shift assays, does not correlate strictly with processing, vice versa (e.g., Figs. 3D, $5,7)$. How to explain this uncoupling? Product release may be a factor; but because hDicer was present in excess over RNA, another possibility is that hDicer binds but does not cleave the RNA readily. Both RNase III-type enzymes and
RNAs undergo induced fit upon binding (Gan et al. 2006, 2008; Wang et al. 2011; Lau et al. 2012). hDicer may need to orient itself properly on a substrate for cleavage to occur, while certain premiRNAs could bind hDicer but fail to induce the necessary conformational changes in the complex or have nucleotides unfavorably aligned near the catalytic center of hDicer. There are such precedents of uncoupling in other RNase III-type enzymes. Yeast RNase III has been suggested to have two distinct modes of RNA binding, one for the formation of a stable RNA/protein complex, and the other leading to RNA cleavage (Lavoie and Abou Elela 2008). $\mathrm{Mg}^{2+}$, absent in our gel shift assays, is essential for the formation of a catalytically competent complex between bacterial RNase III and substrates (Gan et al. 2006, 2008).

Lau et al. (2012) recently proposed a model of hDicer structure based on tagging and electron microscopic studies. Accordingly, the PAZ domain forms the head of an L-shaped hDicer, the RNase III domains reside in the body but near the base, and the helicase domain adopts a clamp shape at the base. If the PAZ domain binds the free ends of a pre-miRNA, then the RNase III domains are well suited to cleave near the stem and terminal loop junction of the pre-miRNA, with a still poorly defined "ruler" domain between the PAZ and RNase domains presumably binding to much of the stem. In this model, the helicase domain is in a good position to contact the terminal loop of a premiRNA, but whether it actually binds the stem, the terminal loop, or both, remains unknown. Future studies are needed to elucidate how the loop is recognized; e.g., how big is the footprint of hDicer binding?

In conclusion, this study investigated the binding and cleavage of pre-miRNAs by hDicer in vitro at a much larger scale than previously reported. The results show that hDicer tolerates significant structural variations in its natural substrates, although certain characteristics, including a 2-nt $3^{\prime}$ overhang, a large terminal loop ( $\geq 10 \mathrm{nt}$ ), and a mismatch-containing stem, favor pre-miRNA cleavage by hDicer.

\section{MATERIALS AND METHODS}

\section{Preparation of RNA substrates}

Sequences and secondary structures of human pre-miRNAs were predicted based on miRBase (Kozomara and Griffiths-Jones 


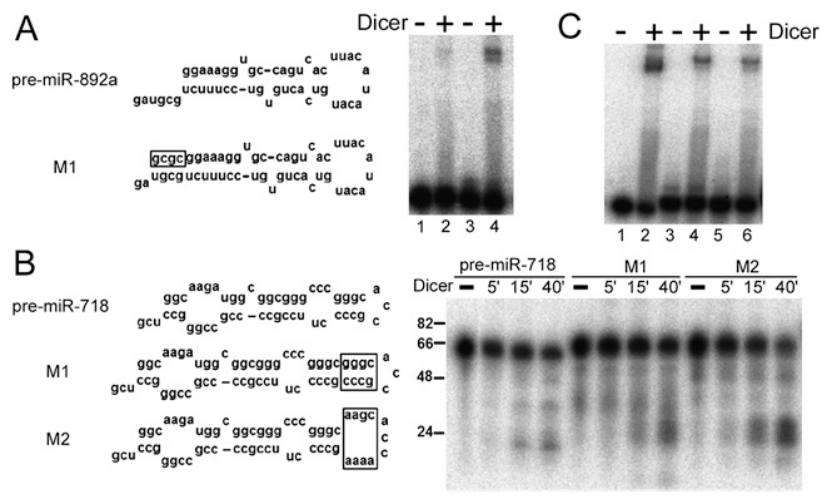

FIGURE 7. Mutations that rescued hDicer binding or cleavage. $(A)$ Gel shift assay of the wild-type pre-miR-892a (lanes 1,2) and its M1 mutant (lanes 3,4). Mutations in M1 are marked with a box. (B) Predicted secondary structures of pre-miR-718 and its M1 and M2 mutants. Mutations are marked with a box. The RNAs were treated with Dicer for 5, 15, and $40 \mathrm{~min}$. Sizes of DNA markers (in nucleotides) are indicated at the left. (C) Gel shift assay. (Lanes 1,2) Wild-type premiR-718; (lanes 3,4) M1; (lanes 5,6) M2. Pre-miR-892a and pre-miR718 and mutants were tested at least twice, and representative results are shown in $A-C$.

2011) and Mfold (Zuker 2003). DNA templates for the wild-type and mutant pre-miRNAs were amplified using PCR, with 5' primers containing the T7 promoter sequence. Because the T7 RNA polymerase prefers transcription initiation from a $G$ residue, if a predicted pre-miRNA does not have a 5' G, we would change it to a $\mathrm{G}$ and make the corresponding change near the $3^{\prime}$ end to maintain the predicted secondary structure. Eightythree percent of the pre-miRNA substrates contained such substitutions (Supplemental Table 1). To prepare ${ }^{32} \mathrm{P}$-labeled substrates, RNAs were produced by in vitro transcription (Promega) in the presence of $\left[\alpha^{32} \mathrm{P}\right] \mathrm{CTP}$. Alternatively, pre-miRNAs were in vitro-transcribed without radioactivity, dephosphorylated, and then labeled with $\left[\gamma_{-}{ }^{32} \mathrm{P}\right]$ ATP using T4 polynucleotide kinase. The first labeling method generates RNA with a $5^{\prime} \mathrm{pppG}$, while the second labels RNA with a $5^{\prime}$ phosphate, present in pre-miRNAs in vivo. It has been shown that the $5^{\prime}$ triphosphate group does not affect hDicer digestion of dsRNAs (Zhang et al. 2002). We also gel-purified pre-miRNAs from Drosha cleavage reactions of internally labeled pri-miRNAs, which is the closest to endogenous pre-miRNAs (Zeng et al. 2005), or labeled chemically synthesized pre-miRNAs (Dharmacon) with $\left[\gamma^{-32} \mathrm{P}\right]$ ATP using T4 polynucleotide kinase. All labeled RNAs were gel-purified before use. RNA yield was calculated, taking into account of the specific activity and half-life of $\left[\alpha-{ }^{32} \mathrm{P}\right] \mathrm{CTP}$ and the number of $\mathrm{C}$ residues in the RNA.

\section{Dicer cleavage assay}

The plasmid encoding hDicer with an N-terminal $3 \times$ FLAG tag, p3 $\times$ FLAG-CMV-Dicer, was constructed by amplifying the Dicer sequence from human cDNA and inserting it into the HindIII and XbaI sites of p3×FLAG-CMV 7.1 (Sigma-Aldrich). Recombinant Dicer was overexpressed and isolated from 293T cells, as described for Drosha (Zhang and Zeng 2010). The concentration of purified hDicer was estimated by SDS-PAGE and Coomassie staining, with known amounts of bovine serum albumin as standards. For processing reactions, a ${ }^{32} \mathrm{P}$-labeled pre-miRNA was mixed with hDicer in a total volume of $6-10 \mu \mathrm{L}$ at $37^{\circ} \mathrm{C}$. The reaction buffer contained $20 \mathrm{mM}$ Tris ( $\mathrm{pH} 7.4$ ), $50 \mathrm{mM} \mathrm{NaCl}$, and $5 \%$ glycerol, supplemented with $\sim 3 \mathrm{mM} \mathrm{MgCl}_{2}, 10 \mathrm{mM} \mathrm{DTT}$, and RNasin (Promega). At specified times, an equal volume of $2 \times$ sample buffer ( $98 \%$ formamide, $10 \mathrm{mM}$ EDTA, and $0.1 \%$ bromophenol blue) was added. After running the samples on a $12 \%$ denaturing polyacrylamide gel and fixing the gel, data were analyzed using a PhosphorImager (GE Healthcare) or by autoradiography. The Dicer cleavage ratio was calculated as the intensities of the product(s) divided by the intensities of the product(s) and remaining substrate. For some pre-miRNAs, their treatment with hDicer also generated singly (partially) digested products. Such intermediates were present at low abundance compared with the fully digested products and, hence, not included in the calculation of Dicer cleavage ratios. Unless stated otherwise, pre-let-7a served as a normalization control in every processing experiment, and the relative cleavage efficiencies of all the premiRNAs in the same experiment were determined by dividing their end-point Dicer cleavage ratios by that of pre-let-7a. For markers, $\phi X 174$ DNA/HinfI (Promega) or synthetic RNA oligonucleotides (Dharmacon) were labeled with $\left[\gamma^{-32} \mathrm{P}\right]$ ATP using T4 polynucleotide kinase.

\section{Gel shift assay}

hDicer was incubated with a ${ }^{32} \mathrm{P}$-labeled RNA in $20 \mathrm{mM}$ Tris $(\mathrm{pH}$ 7.4), $50 \mathrm{mM} \mathrm{KCl}, 0.1 \%$ Tween $20,0.5 \mathrm{mM}$ EDTA, and $10 \%$ glycerol on ice for $20-60 \mathrm{~min}$. Then $1 \mu \mathrm{L}$ of $1 \%$ bromophenol blue was added, and samples were run on a $7.5 \%$ native polyacrylamide gel. After fixation, the gel was exposed to a PhosphorImager screen or an X-ray film. Binding ratio or efficiency was calculated as the intensity of the shifted species divided by the intensity of the free RNA and the shifted species.

\section{Nuclease protection assays}

For RNase V1 (Invitrogen) digestion, ${ }^{32} \mathrm{P}$-labeled RNAs were diluted in $20 \mathrm{mM}$ Tris ( $\mathrm{pH} 7.4$ ), $50 \mathrm{mM} \mathrm{NaCl}, 5 \%$ glycerol, and $0.5 \mathrm{mM}$ EDTA, and incubated with hDicer in a total volume of $4 \mu \mathrm{L}$ on ice for $\sim 20 \mathrm{~min}$. RNase V1 (0.001-0.004 units) in $2 \mu \mathrm{L}$ of $3 \times$ RNA structure buffer (Invitrogen) was then added, and incubation continued at room temperature. The final concentration of RNAs was $\sim 1 \mathrm{nM}$, and hDicer $\sim 30 \mathrm{nM}$. At specified times, $6 \mu \mathrm{L}$ of $2 \times$ sample buffer was added. After running the samples on a $12 \%$ denaturing gel, data were analyzed using a PhosphorImager. For $\mathrm{S} 1$ nuclease (Promega) digestion, $5^{\prime}$-end ${ }^{32} \mathrm{P}$-labeled RNAs were diluted in $1 \times \mathrm{S} 1$ buffer (Promega) and mixed with bovine serum albumin or hDicer on ice. S1 nuclease was then added at 3 units per reaction, and incubation continued at room temperature for $10 \mathrm{~min}$. Products were resolved by gel electrophoresis and analyzed using a PhosphorImager.

\section{Statistics analysis}

Averages \pm standard deviations are presented in the text and figures where $n \geq 3$. GraphPad Prism 5.0 (GraphPad Software) was used to calculate Spearman rank correlation coefficients, with the $P$-values computed by a permutation test, and the $P$-values in a Mann-Whitney U-test. 


\section{SUPPLEMENTAL MATERIAL}

Supplemental material is available for this article.

Received April 5, 2012; accepted August 7, 2012.

\section{REFERENCES}

Bartel DP. 2004. MicroRNAs: Genomics, biogenesis, mechanism, and function. Cell 116: 281-297.

Chakravarthy S, Sternberg SH, Kellenberger CA, Doudna JA. 2010. Substrate-specific kinetics of Dicer-catalyzed RNA processing. J Mol Biol 404: 392-402.

Du Z, Lee JK, Tjhen R, Stroud RM, James TL. 2008. Structural and biochemical insights into the dicing mechanism of mouse Dicer: A conserved lysine is critical for dsRNA cleavage. Proc Natl Acad Sci 105: 2391-2396.

Feng Y, Zhang X, Song Q, Li T, Zeng Y. 2011. Drosha processing controls the specificity and efficiency of global microRNA expression. Biochim Biophys Acta 1809: 700-707.

Gan J, Tropea JE, Austin BP, Court DL, Waugh DS, Ji X. 2006. Structural insight into the mechanism of double-stranded RNA processing by ribonuclease III. Cell 124: $355-366$.

Gan J, Shaw G, Tropea JE, Waugh DS, Court DL, Ji X. 2008. A stepwise model for double-stranded RNA processing by ribonuclease III. Mol Microbiol 67: 143-154.

Koscianska E, Starega-Roslan J, Krzyzosiak WJ. 2011. The role of Dicer protein partners in the processing of microRNA precursors. PLoS ONE 6: e28548. doi: 10.1371/journal.pone.0028548.

Kozomara A, Griffiths-Jones S. 2011. miRBase: Integrating microRNA annotation and deep-sequencing data. Nucleic Acids Res 39: D152D157.

Landgraf P, Rusu M, Sheridan R, Sewer A, Iovino N, Aravin A, Pfeffer S, Rice A, Kamphorst AO, Landthaler M, et al. 2007. A mammalian microRNA expression atlas based on small RNA library sequencing. Cell 129: 1401-1414.

Lau PW, Guiley KZ, De N, Potter CS, Carragher B. 2012. The molecular architecture of human Dicer. Nat Struct Mol Biol 19: 436-440.

Lavoie M, Abou Elela S. 2008. Yeast ribonuclease III uses a network of multiple hydrogen bonds for RNA binding and cleavage. Biochemistry 47: 8514-8526.

Lee YS, Nakahara K, Pham JW, Kim K, He Z, Sontheimer EJ, Carthew RW. 2004. Distinct roles for Drosophila Dicer-1 and Dicer-2 in the siRNA/miRNA silencing pathways. Cell 117: 69-81.

Ma JB, Ye K, Patel DJ. 2004. Structural basis for overhang-specific small interfering RNA recognition by the PAZ domain. Nature 429: $318-322$.
Ma E, MacRae IJ, Kirsch JF, Doudna JA. 2008. Autoinhibition of human Dicer by its internal helicase domain. J Mol Biol 380: 237-243.

MacRae IJ, Zhou K, Li F, Repic A, Brooks AN, Cande WZ, Adams PD, Doudna JA. 2006. Structural basis for double-stranded RNA processing by Dicer. Science 311: 195-198.

MacRae IJ, Zhou K, Doudna JA. 2007. Structural determinants of RNA recognition and cleavage by Dicer. Nat Struct Mol Biol 14: 934-940.

Milligan JF, Groebe DR, Witherell GW, Uhlenbeck OC. 1987. Oligoribonucleotide synthesis using T7 RNA polymerase and synthetic DNA templates. Nucleic Acids Res 15: 8783-8798.

Park JE, Heo I, Tian Y, Simanshu DK, Chang H, Jee D, Patel DJ, Kim VN. 2011. Dicer recognizes the $5^{\prime}$ end of RNA for efficient and accurate processing. Nature 475: 201-205.

Provost P, Dishart D, Doucet J, Frendewey D, Samuelsson B, Radmark O. 2002. Ribonuclease activity and RNA binding of recombinant human Dicer. EMBO J 21: 5864-5874.

Sashital DG, Doudna JA. 2010. Structural insights into RNA interference. Curr Opin Struct Biol 20: 90-97.

Starega-Roslan J, Krol J, Koscianska E, Kozlowski P, Szlachcic WJ, Sobczak K, Krzyzosiak WJ. 2011. Structural basis of microRNA length variety. Nucleic Acids Res 39: 257-268.

Tsutsumi A, Kawamata T, Izumi N, Seitz H, Tomari Y. 2011. Recognition of the pre-miRNA structure by Drosophila Dicer-1. Nat Struct Mol Biol 18: 1153-1158.

Vermeulen A, Behlen L, Reynolds A, Wolfson A, Marshall WS, Karpilow J, Khvorova A. 2005. The contributions of dsRNA structure to Dicer specificity and efficiency. RNA 11: 674-682.

Wang Z, Hartman E, Roy K, Chanfreau G, Feigon J. 2011. Structure of a yeast RNase III dsRBD complex with a noncanonical RNA substrate provides new insights into binding specificity of dsRBDs. Structure 19: 999-1010.

Welker NC, Maity TS, Ye X, Aruscavage PJ, Krauchuk AA, Liu Q, Bass BL. 2011. Dicer's helicase domain discriminates dsRNA termini to promote an altered reaction mode. Mol Cell 41: 589-599.

Zeng Y, Yi R, Cullen BR. 2005. Recognition and cleavage of primary microRNA precursors by the nuclear processing enzyme Drosha. EMBO J 24: 138-148.

Zhang X, Zeng Y. 2010. The terminal loop region controls microRNA processing by Drosha and Dicer. Nucleic Acids Res 38: 76897697.

Zhang H, Kolb FA, Brondani V, Billy E, Filipowicz W. 2002. Human Dicer preferentially cleaves dsRNAs at their termini without a requirement for ATP. EMBO J 21: 5875-5885.

Zhang H, Kolb FA, Jaskiewicz L, Westhof E, Filipowicz W. 2004. Single processing center models for human Dicer and bacterial RNase III. Cell 118: 57-68.

Zuker M. 2003. Mfold web server for nucleic acid folding and hybridization prediction. Nucleic Acids Res 31: 3406-3415. 

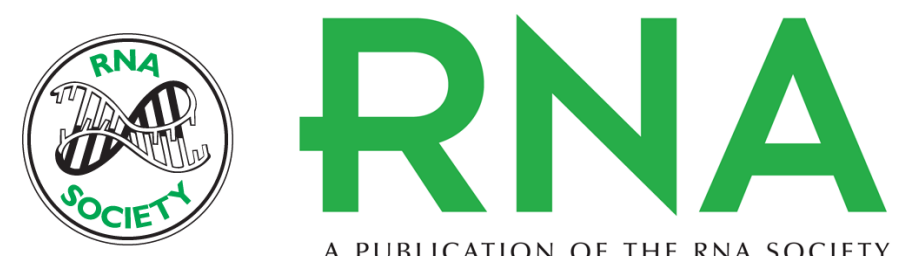

A PUBLICATION OF THE RNA SOCIETY

\section{A comprehensive analysis of precursor microRNA cleavage by human Dicer}

Yong Feng, Xiaoxiao Zhang, Paul Graves, et al.

RNA 2012 18: 2083-2092 originally published online September 14, 2012

Access the most recent version at doi:10.1261/rna.033688.112

Supplemental http://rnajournal.cshlp.org/content/suppl/2012/09/04/rna.033688.112.DC1
Material

References This article cites 30 articles, 6 of which can be accessed free at:

http://rnajournal.cshlp.org/content/18/11/2083.full.html\#ref-list-1

\section{License}

Email Alerting Receive free email alerts when new articles cite this article - sign up in the box at the Service top right corner of the article or click here. 